\title{
Coherent synchrotron radiation instability in a bunch compressor
}

\author{
S. Heifets and G. Stupakov \\ Stanford Linear Accelerator Center, Stanford University, Stanford, California 94309 \\ S. Krinsky \\ NSLS, Brookhaven National Laboratory, Upton, New York 11973
}

(Received 19 March 2002; published 5 June 2002)

\begin{abstract}
The coherent synchrotron radiation of a bunch in a bunch compressor may lead to the microwave instability producing longitudinal modulation of the bunch with wavelengths small compared to the bunch length. It can also be a source of an undesirable emittance growth in the compressor. We derive and analyze the equation that describes linear evolution of the microwave modulation taking into account incoherent energy spread and finite emittance of the beam. Numerical solution of this equation for the Linac Coherent Light Source bunch compressor gives the amplification factor for different wavelengths of the beam microbunching.
\end{abstract}

DOI: 10.1103/PhysRevSTAB.5.064401

PACS numbers: 29.27.-a

\section{INTRODUCTION}

The design of a magnetic bunch compressor chicane is a key technical challenge in the development of linac driven $\mathrm{x}$-ray free-electron lasers. Compression is essential to reduce the length of the electron bunch and correspondingly to increase its peak current. In order to achieve compression, a relativistic electron bunch is given an energy chirp while passing through linac sections off crest. Since higher energy electrons follow a shorter trajectory through the chicane, the high energy electrons at the back of the bunch catch up with the lower energy electrons in the front. A major concern in the design is to assure that bunch compression does not degrade the quality of the electron beam. In particular, it is important not to increase the energy spread or transverse emittance beyond acceptable tolerances.

The beam in the chicane can radiate coherently if the wavelength of the radiation exceeds the length of the bunch. This radiation results in an undesirable growth of the beam emittance [1], which can, however, be cured (at least partially) by a special design of the compressor [2].

As was pointed out in Ref. [3], coherent synchrotron radiation (CSR) can also be a source of modulation of the beam density at wavelengths small compared to the bunch length. The results of Ref. [3] refer to the microbunching instability in a ring; however, a similar effect can also occur in a bunch compressor where coherent synchrotron radiation often plays a role. Indeed, the effect of microbunching caused by CSR has been observed in computer simulations of the bunch compressor [4] designed for the Linac Coherent Light Source (LCLS) at SLAC [5], and also in the simulations of the TESLA Test Facility bunch compressor [6]. Analytical estimates of the CSR effects in bunch compressors have been published in Ref. [7].

An approach to treat the CSR induced microbunching in the case when the beam is being compressed in the magnetic chicane has been developed in [8]. Reference [8] used an oversimplified one-dimensional model which does not take properly into account the transverse motion of the beam. The importance of the correct account of this motion has been recently pointed out in Ref. [9], where the microbunching of the beam is considered as due to the klystron instability under the influence of the CSR wakefield. However, the treatment in Ref. [9] is valid only in the absence of the energy chirp, and hence neglects the effect of compression on the instability.

In this paper, we discuss the amplification of a highfrequency density modulation of the electron beam in a bunch compressor. The effect of the beam compression on the microbunching is taken into account. The high frequency means that the wavelength of the disturbance is short compared to the electron bunch length. We assume that the shielding effect of the conducting walls on CSR is not important, which is usually true when the bunch length is short enough. We will also neglect the transient effects in the CSR wake [10] occurring in short magnets.

Our analysis is based upon a linearized Vlasov equation that includes longitudinal and radial degrees of freedom. It is necessary to take into account the radial degrees of freedom since the variation of the path length with energy in the chicane results from the transverse dispersion of the trajectory.

In order to solve the linear Vlasov partial differential equation, we use the method of characteristics to reduce it to a linear Volterra integral equation for the Fourier component of the density modulation. The integral equation provides a description of the microwave instability as well as klystron-type amplification. Landau damping due to the electron beam energy spread and transverse emittance is included in our analysis.

The paper is organized as follows. In Sec. II we introduce optical functions in the compressor, solve equations of motion, and find integrals of motion for the particle. 
The equilibrium distribution function of the beam with the energy chirp is introduced in Sec. III as a solution of the Vlasov equation. The CSR wake term is added to the Vlasov equation in Sec. IV, and the equation is linearized, assuming that the perturbation of the equilibrium distribution function is small. Integrating the linearized Vlasov equation along the unperturbed trajectories in Sec. V, we reduce it to a one-dimensional Volterra integral equation. In Sec. VI we present the results of numerical solution of the integral equation for the LCLS bunch compressor. In the last section, Sec. VII, the results of the paper are summarized.

\section{OPTICS OF BUNCH COMPRESSOR}

Let us consider, first, single particle motion in a bunch compressor. We use the notation $x$ for the horizontal offset of a particle relative to the nominal orbit, $\theta=d x / d s$ is the angular slope of the orbit, $p=\Delta E / E$ is the relative energy deviation of the particle, $z$ is the longitudinal coordinate of the particle in the bunch, and $s$ is the path length along the nominal orbit.

Equations for $x, \theta, z$, and $p$ have the form

$$
\begin{gathered}
\frac{d x}{d s}=\theta, \quad \frac{d \theta}{d s}=-k_{\beta}(s)^{2} x+\frac{p}{R(s)}, \\
\frac{d z}{d s}=-\frac{x}{R(s)}, \quad \frac{d p}{d s}=0,
\end{gathered}
$$

where $R(s)$ is the bending radius in dipole magnets and $k_{\beta}(s)$ is the focusing strength. Typically, quadrupole magnets are not used in bunch compressors; however, for the sake of generality, we include the focusing term in Eqs. (1). These equations have a formal solution

$$
\begin{aligned}
x= & D p+\sqrt{\beta}\left(\frac{x_{0}}{\sqrt{\beta_{0}}} \cos \psi+\theta_{0} \sqrt{\beta_{0}} \sin \psi\right), \\
\theta= & D^{\prime} p-\frac{\alpha}{\beta}(x-D p) \\
& -\frac{1}{\sqrt{\beta}}\left(\frac{x_{0}}{\sqrt{\beta_{0}}} \sin \psi-\theta_{0} \sqrt{\beta_{0}} \cos \psi\right), \\
z= & z_{0}+R_{56} p+x_{0} R_{51}+\theta_{0} R_{52},
\end{aligned}
$$

where $x_{0}, \theta_{0}$, and $z_{0}$ are constants given by initial conditions, $\beta(s) \equiv w(s)^{2}$ is the beta function, $\beta_{0}$ is the initial value of the beta function at the entrance to the compressor, $\beta_{0}=\beta(0), \alpha(s)=-(1 / 2) d \beta / d s, D(s)$ is the dispersion function, $D^{\prime}(s)=d D / d s$, and $w(s)$ and $D(s)$ satisfy the following equations:

$$
\begin{aligned}
& w^{\prime \prime}+k_{\beta}^{2} w=\frac{1}{w^{3}}, \\
& D^{\prime \prime}+k_{\beta}^{2} D=\frac{1}{R} .
\end{aligned}
$$

The functions $R_{51}, R_{52}, R_{56}$, and the betatron phase $\psi$ can be computed by integration [11]

$$
\begin{aligned}
\psi(s) & =\int_{0}^{s} \frac{d s^{\prime}}{\beta\left(s^{\prime}\right)}, \\
R_{56}(s) & =-\int_{0}^{s} \frac{D\left(s^{\prime}\right)}{R\left(s^{\prime}\right)} d s^{\prime}, \\
R_{51}(s) & =-\frac{1}{\sqrt{\beta_{0}}} \int_{0}^{s} \frac{\sqrt{\beta\left(s^{\prime}\right)}}{R\left(s^{\prime}\right)} \cos \psi\left(s^{\prime}\right) d s^{\prime}, \\
R_{52}(s) & =-\sqrt{\beta_{0}} \int_{0}^{s} \frac{\sqrt{\beta\left(s^{\prime}\right)}}{R\left(s^{\prime}\right)} \sin \psi\left(s^{\prime}\right) d s^{\prime} .
\end{aligned}
$$

We assume that the dispersion function and its derivative, by design, have zero initial values $D(0)=D^{\prime}(0)=0$. In this case, $D$ is given by the following equation:

$$
D(s)=\sqrt{\beta(s)} \int_{0}^{s} \frac{d s^{\prime}}{R\left(s^{\prime}\right)} \sqrt{\beta\left(s^{\prime}\right)} \sin \left[\psi(s)-\psi\left(s^{\prime}\right)\right] .
$$

The parameters $x_{0}, \theta_{0}$, and $z_{0}$ are the constants of motion. They determine the amplitude and phase of the betatron oscillations and can be expressed as functions of current coordinates of the particle $x, \theta$, and $p$ at position $s$,

$$
\begin{aligned}
& x_{0}(x, \theta, p, s)=\sqrt{\frac{\beta_{0}}{\beta}}(x-D p) \cos \psi-\sqrt{\beta_{0} \beta}\left[\theta-D^{\prime} p+\frac{\alpha}{\beta}(x-D p)\right] \sin \psi, \\
& \theta_{0}(x, \theta, p, s)=\frac{x-D p}{\sqrt{\beta \beta_{0}}} \sin \psi+\sqrt{\frac{\beta}{\beta_{0}}}\left[\theta-D^{\prime} p+\frac{\alpha}{\beta}(x-D p)\right] \cos \psi, \\
& z_{0}(x, \theta, p, s)=z-R_{56} p-x_{0} R_{51}-\theta_{0} R_{52} .
\end{aligned}
$$

Note that Eqs. (6) constitute a canonical transformation from the variables $x, \theta, z, p$ to $x_{0}, \theta_{0}, z_{0}, p$, with the Jacobian of the transformation equal to unity. 


\section{DISTRIBUTION FUNCTION OF THE BEAM IN BUNCH COMPRESSOR}

We define the distribution function $\rho(x, \theta, z, p, s)$ of the beam in a bunch compressor so that the integral $\int d x d \theta d p \rho$ gives the number of particles per unit length of the beam. Neglecting the wakefield effects, the equilibrium distribution function $\rho_{0}(x, \theta, z, p, s)$ satisfies the Vlasov equation,

$$
\frac{\partial \rho_{0}}{\partial s}-\frac{x}{R} \frac{\partial \rho_{0}}{\partial z}+\theta \frac{\partial \rho_{0}}{\partial x}+\left(-k_{\beta}^{2} x+\frac{p}{R}\right) \frac{\partial \rho_{0}}{\partial \theta}=0
$$

Equations (6) are characteristics of Eq. (7). Therefore, the solution of Eq. (7) is an arbitrary function of the integrals of motion

$$
\rho_{0}(x, \theta, z, p, s)=f_{0}\left[x_{0}(x, \theta, z, p, s), \theta_{0}(x, \theta, z, p, s), z_{0}(x, \theta, z, p, s), p\right] .
$$

For the equilibrium function $f_{0}$ we choose a model of a coasting beam with Gaussian distributions over the initial coordinates $x_{0}$ and $\theta_{0}$ as well as over $p$,

$$
f_{0}=\frac{n_{b}}{2 \pi \epsilon_{0}} \exp \left(-\frac{x_{0}^{2}+\left(\beta_{0} \theta_{0}\right)^{2}}{2 \epsilon_{0} \beta_{0}}\right) \rho_{G}\left(p+u z_{0}\right),
$$

where

$$
\rho_{G}(p)=\frac{1}{\sqrt{2 \pi} \sigma_{p}} \exp \left(-\frac{p^{2}}{2 \sigma_{p}^{2}}\right)
$$

$n_{b}$ is the number of particles per unit length of the beam at the compressor entrance $(s=0), \epsilon_{0}$ is the horizontal emittance, and $\sigma_{p}$ is the uncorrelated energy spread of the beam. The chirp parameter $u$ in this equation accounts for the correlation between the position of the particle in the bunch and its energy. This correlation is generated by an acceleration section at the entrance to the compressor. For negative values of $R_{56}$, the chirp $u<0$ leads to the compression of the bunch.

Note that in this model the linear density of the beam $\int d x d \theta d p \rho_{0}$ does not depend on $z$, in agreement with the fact that $\rho_{0}$ describes a coasting beam.

\section{VLASOV EQUATION WITH THE CSR WAKE}

Let us now take into account the CSR wake of the beam. It can be described by the wake function $W(z, s)$ such that $W \neq 0$ for $z>0[12,13]$. For our purposes it is convenient to introduce the CSR impedance $Z(k, s)$,

$$
Z(k, s)=\int_{0}^{\infty} d \zeta W(\zeta, s) e^{-i k \zeta}=-\frac{i k^{1 / 3} A}{R(s)^{2 / 3}},
$$

where

$$
A=3^{-1 / 3} \Gamma\left(\frac{2}{3}\right)(\sqrt{3} i-1)=1.63 i-0.94,
$$

with $\Gamma$ the complete gamma function. In Eq. (11) and below we use the cgs system of units. Using Eq. (11) we assume that the retardation time for the coherent radiation is small compared to the length of the bend and neglect the formation length of the radiation in comparison with the length of the magnet [10].

The Vlasov equation for the distribution function $\rho(x, \theta, z, p, s)$ including the wake takes the form:

$$
\begin{array}{r}
\frac{\partial \rho}{\partial s}-\frac{x}{R} \frac{\partial \rho}{\partial z}+\theta \frac{\partial \rho}{\partial x}+\left(-k_{\beta}^{2} x+\frac{p}{R}\right) \frac{\partial \rho}{\partial \theta} \\
=\frac{r_{e}}{\gamma} \frac{\partial \rho}{\partial p} \int d z^{\prime} W\left(z-z^{\prime}, s\right) n\left(z^{\prime}, s\right),
\end{array}
$$

where $n(z, s)=\int d x d \theta d p \rho(x, \theta, z, p, s)$.

Note that the equilibrium distribution function $\rho_{0}$, which we found as a solution of Eq. (7), also satisfies Eq. (13), because the equilibrium beam density $n_{0}(z, s)$, where

$$
\begin{aligned}
n_{0}(z, s) & =\int d x d \theta d p \rho_{0} \\
& =\int d x_{0} d \theta_{0} d p f_{0} \\
& =\frac{n_{b}}{1-u R_{56}},
\end{aligned}
$$

is independent of $z$, and $\int d z^{\prime} W\left(z-z^{\prime}\right) n_{0}\left(z^{\prime}, s\right)=0$ [in Eq. (14) we changed variables using the invariance of the phase volume $d x d \theta=d x_{0} d \theta_{0}$ ].

Let us consider a small perturbation of the equilibrium distribution function

$$
\rho=\rho_{0}+\rho_{1},
$$

where $\rho_{1} \ll \rho_{0}$. The linear stability of the beam is defined by the linearized Vlasov equation for $\rho_{1}$ which takes the form

$$
\begin{array}{r}
\frac{\partial \rho_{1}}{\partial s}-\frac{x}{R} \frac{\partial \rho_{1}}{\partial z}+\theta \frac{\partial \rho_{1}}{\partial x}+\left(-k_{\beta}^{2} x+\frac{p}{R}\right) \frac{\partial \rho_{1}}{\partial \theta} \\
=\frac{r_{e}}{\gamma} \frac{\partial \rho_{0}}{\partial p} \int d z^{\prime} W\left(z-z^{\prime}, s\right) n_{1}\left(z^{\prime}, s\right),
\end{array}
$$

where $n_{1}(z, s)=\int d x d \theta d p \rho_{1}(x, \theta, z, p, s)$. 
We will seek solution of Eq. (16) as a function of the invariants $x_{0}, \theta_{0}, z_{0}$, and also $p$ and $s$ :

$$
\rho_{1}(x, \theta, z, p, s)=f_{1}\left(x_{0}, \theta_{0}, z_{0}, p, s\right),
$$

where $x_{0}, \theta_{0}$, and $z_{0}$ are the functions given by Eqs. (6). The function $f_{1}$ satisfies the following equation:

$$
\begin{aligned}
\frac{\partial f_{1}}{\partial s}= & \frac{r_{e}}{\gamma} \frac{\partial \rho_{0}}{\partial p} \int d z^{\prime} \\
& \times W\left(z_{0}+p R_{56}+x_{0} R_{51}+\theta_{0} R_{52}-z^{\prime}, s\right) \\
& \times n_{1}\left(z^{\prime}, s\right) .
\end{aligned}
$$

Here the derivative $\partial \rho_{0} / \partial p$ is understood as a function of variables $x_{0}, \theta_{0}, z_{0}$, and $s$ and can be calculated using Eqs. (8) and (9),

$$
\begin{array}{r}
\frac{\partial \rho_{0}}{\partial p}=-f_{0}\left[\frac{x_{0}}{\epsilon_{0} \beta_{0}} \frac{\partial x_{0}}{\partial p}+\frac{\beta_{0} \theta_{0}}{\epsilon_{0}} \frac{\partial \theta_{0}}{\partial p}\right. \\
\left.+\frac{p+u z_{0}}{\sigma_{p}^{2}}\left(1+u \frac{\partial z_{0}}{\partial p}\right)\right],
\end{array}
$$

where the derivatives

$$
\begin{aligned}
& \frac{\partial x_{0}}{\partial p}=-\sqrt{\frac{\beta_{0}}{\beta}} D \cos \psi+\sqrt{\beta \beta_{0}}\left(D^{\prime}+\frac{\alpha}{\beta} D\right) \sin \psi, \\
& \frac{\partial \theta_{0}}{\partial p}=-\frac{D}{\sqrt{\beta \beta_{0}}} \sin \psi-\sqrt{\frac{\beta}{\beta_{0}}}\left(D^{\prime}+\frac{\alpha}{\beta} D\right) \cos \psi,
\end{aligned}
$$

$\frac{\partial z_{0}}{\partial p}=-R_{56}-R_{51} \frac{\partial x_{0}}{\partial p}-R_{52} \frac{\partial \theta_{0}}{\partial p}$

are functions of $s$ only.

The density perturbation $n_{1}$ can be calculated by changing the integration variables and introducing an additional $\delta$ function in the integrand:

$$
\begin{aligned}
n_{1}(z, s)= & \int d x d \theta d p \rho_{1} \\
= & \int d x_{0} d \theta_{0} d z_{0} d p f_{1}\left(x_{0}, \theta_{0}, z_{0}, p, s\right) \\
& \times \delta\left(z_{0}+R_{56} p+x_{0} R_{51}+\theta_{0} R_{52}-z\right) .
\end{aligned}
$$

Substituting Eqs. (19) and (21) into Eq. (18) we obtain the following equation for $f_{1}$ :

$$
\begin{aligned}
\frac{\partial f_{1}}{\partial s}= & -\frac{r_{e}}{\gamma} f_{0}\left[\frac{x_{0}}{\epsilon_{0} \beta_{0}} \frac{\partial x_{0}}{\partial p}+\frac{\beta_{0} \theta_{0}}{\epsilon_{0}} \frac{\partial \theta_{0}}{\partial p}+\frac{p+u z_{0}}{\sigma_{p}^{2}}\left(1+u \frac{\partial z_{0}}{\partial p}\right)\right] \\
& \times \int d x_{0}^{\prime} d \theta_{0}^{\prime} d z_{0}^{\prime} d p^{\prime} f_{1}\left(x_{0}^{\prime}, \theta_{0}^{\prime}, z_{0}^{\prime}, p^{\prime}, s\right) W\left[\left(z_{0}-z_{0}^{\prime}+\left(p-p^{\prime}\right) R_{56}+\left(x_{0}-x_{0}^{\prime}\right) R_{51}+\left(\theta_{0}-\theta_{0}^{\prime}\right) R_{52}, s\right] .\right.
\end{aligned}
$$

It is convenient to use a new variable $p_{0}=p+u z_{0}$ instead of $p$ and consider $f_{0}$ as a function of $x_{0}, \theta_{0}$, and $p_{0}, f_{0}=$ $f_{0}\left(x_{0}, \theta_{0}, p_{0}\right)$, and $f_{1}$ as a function of variables $x_{0}, \theta_{0}, z_{0}, p_{0}$, and $s, f_{1}=f_{1}\left(x_{0}, \theta_{0}, z_{0}, p_{0}, s\right)$. Note that the transformation from $x, \theta, z$, and $p$ to $x_{0}, \theta_{0}, z_{0}$, and $p_{0}$ is canonical and has a unit Jacobian. Then, Eq. (22) takes the form

$$
\begin{aligned}
\frac{\partial f_{1}}{\partial s}= & -\frac{r_{e}}{\gamma} f_{0}\left[\frac{x_{0}}{\epsilon_{0} \beta_{0}} \frac{\partial x_{0}}{\partial p}+\frac{\beta_{0} \theta_{0}}{\epsilon_{0}} \frac{\partial \theta_{0}}{\partial p}+\frac{p_{0}}{\sigma_{p}^{2}}\left(1+u \frac{\partial z_{0}}{\partial p}\right)\right] \\
& \times \int d x_{0}^{\prime} d \theta_{0}^{\prime} d z_{0}^{\prime} d p_{0}^{\prime} f_{1}\left(x_{0}^{\prime}, \theta_{0}^{\prime}, z_{0}^{\prime}, p_{0}^{\prime}, s\right) \\
& \times W\left[\left(z_{0}-z_{0}^{\prime}\right)\left(1-u R_{56}\right)+\left(p_{0}-p_{0}^{\prime}\right) R_{56}+\left(x_{0}-x_{0}^{\prime}\right) R_{51}+\left(\theta_{0}-\theta_{0}^{\prime}\right) R_{52}, s\right] .
\end{aligned}
$$

\section{SOLUTION OF THE LINEARIZED VLASOV EQUATION}

Now we will assume a sinusoidal dependence of $f_{1}$ versus $z_{0}$,

$$
f_{1}\left(x_{0}, \theta_{0}, z_{0}, p_{0}, s\right)=f_{k}\left(x_{0}, \theta_{0}, p_{0}, s\right) e^{i k z_{0}} .
$$

Substituting Eq. (24) into Eq. (23) and using definition of the impedance Eq. (11) casts the integral in the left-hand side to the following:

$$
\begin{aligned}
& \int d x_{0}^{\prime} d \theta_{0}^{\prime} d p_{0}^{\prime} f_{k}\left(x_{0}^{\prime}, \theta_{0}^{\prime}, p_{0}^{\prime}, s\right) \int d z_{0}^{\prime} e^{i k z_{0}^{\prime}} W\left[\left(z_{0}-z_{0}^{\prime}\right)\left(1-u R_{56}\right)+\left(p_{0}-p_{0}^{\prime}\right) R_{56}\right. \\
& \left.+\left(x_{0}-x_{0}^{\prime}\right) R_{51}+\left(\theta_{0}-\theta_{0}^{\prime}\right) R_{52}, s\right] \\
& =C(s) Z(k C(s), s) \int d x_{0}^{\prime} d \theta_{0}^{\prime} d p_{0}^{\prime} f_{k}\left(x_{0}^{\prime}, \theta_{0}^{\prime}, p_{0}^{\prime}, s\right) \\
& \times \exp \left\{i k C(s)\left[z_{0}\left(1-u R_{56}\right)+\left(p_{0}-p_{0}^{\prime}\right) R_{56}+\left(x_{0}-x_{0}^{\prime}\right) R_{51}+\left(\theta_{0}-\theta_{0}^{\prime}\right) R_{52}\right]\right\},
\end{aligned}
$$


where we introduced the compression factor $C(s)$ :

$$
C(s)=\frac{1}{1-u R_{56}(s)} .
$$

It is easy to see that the factor $\exp \left(i k z_{0}\right)$ will cancel out in the left- and right-hand sides of Eq. (23), and the resulting equation can be written as follows:

$$
\begin{aligned}
\frac{\partial f_{k}}{\partial s}= & -\left.\frac{r_{e}}{\gamma} f_{0} C(s) Z(k C(s), s)\left[\frac{x_{0}}{\epsilon_{0} \beta_{0}} \frac{\partial x_{0}}{\partial p}+\frac{\beta_{0} \theta_{0}}{\epsilon_{0}} \frac{\partial \theta_{0}}{\partial p}+\frac{p+u z_{0}}{\sigma_{p}^{2}}\left(1+u \frac{\partial z_{0}}{\partial p}\right)\right]\right|_{s} \\
& \times g_{k}(s) \exp \left\{i k C(s)\left[p_{0} R_{56}(s)+x_{0} R_{51}(s)+\theta_{0} R_{52}(s)\right]\right\},
\end{aligned}
$$

where

$$
g_{k}(s)=\int d x_{0} d \theta_{0} d p_{0} f_{k}\left(x_{0}, \theta_{0}, p_{0}, s\right) \exp \left\{-i k C(s)\left[p_{0} R_{56}(s)+x_{0} R_{51}(s)+\theta_{0} R_{52}(s)\right]\right\} .
$$

From Eqs. (21), (24), and (26), we see that $g_{k}(s)$ is related to the amplitude of the density perturbation with the wave number $k$,

$$
n_{1, k}(z, s)=C(s) g_{k}(s) e^{i k C(s) z}
$$

The solution of Eq. (27) can be written as an integral of the right-hand side with an initial condition $f_{k}\left(x_{0}, \theta_{0}, p_{0}, 0\right)$. Substituting this solution in Eq. (28) we obtain the integral equation

$$
g_{k}(s)=g_{k}^{(0)}(s)+\int_{0}^{s} K\left(s, s^{\prime}\right) g_{k}\left(s^{\prime}\right) d s^{\prime},
$$

where the kernel $K$ is

$$
\begin{aligned}
K\left(s, s^{\prime}\right)= & -\left.\frac{r_{e}}{\gamma} C\left(s^{\prime}\right) Z\left(k C\left(s^{\prime}\right), s^{\prime}\right) \int d x_{0} d \theta_{0} d p_{0} f_{0}\left(x_{0}, \theta_{0}, p_{0}\right)\left[\frac{x_{0}}{\epsilon_{0} \beta_{0}} \frac{\partial x_{0}}{\partial p}+\frac{\beta_{0} \theta_{0}}{\epsilon_{0}} \frac{\partial \theta_{0}}{\partial p}+\frac{p+u z_{0}}{\sigma_{p}^{2}}\left(1+u \frac{\partial z_{0}}{\partial p}\right)\right]\right|_{s^{\prime}} \\
& \times \exp \left\{-i k\left[p_{0} R_{56}\left(s, s^{\prime}\right)+x_{0} R_{51}\left(s, s^{\prime}\right)+\theta_{0} R_{52}\left(s, s^{\prime}\right)\right]\right\}
\end{aligned}
$$

and

$$
\begin{aligned}
& R_{51}\left(s, s^{\prime}\right)=C(s) R_{51}(s)-C\left(s^{\prime}\right) R_{51}\left(s^{\prime}\right), \\
& R_{52}\left(s, s^{\prime}\right)=C(s) R_{52}(s)-C\left(s^{\prime}\right) R_{52}\left(s^{\prime}\right), \\
& R_{56}\left(s, s^{\prime}\right)=C(s) R_{56}(s)-C\left(s^{\prime}\right) R_{56}\left(s^{\prime}\right) .
\end{aligned}
$$

The function $g_{k}^{(0)}(s)$ is related to the initial value of $f_{k}$ :

$$
g_{k}^{(0)}(s)=\int d x_{0} d \theta_{0} d p_{0} f_{k}\left(x_{0}, \theta_{0}, p_{0}, 0\right) e^{-i k C(s)\left[p_{0} R_{56}(s)+x_{0} R_{51}(s)+\theta_{0} R_{52}(s)\right]} .
$$

The integrals over $x_{0}$ and $\theta_{0}$ in Eq. (31) can be calculated explicitly,

$$
\begin{gathered}
\left.\int d x_{0} d \theta_{0} f_{0}\left(x_{0}, \theta_{0}, p_{0}\right)\left[\frac{x_{0}}{\epsilon_{0} \beta_{0}} \frac{\partial x_{0}}{\partial p}+\frac{\beta_{0} \theta_{0}}{\epsilon_{0}} \frac{\partial \theta_{0}}{\partial p}+\frac{p+u z_{0}}{\sigma_{p}^{2}}\left(1+u \frac{\partial z_{0}}{\partial p}\right)\right]\right|_{s^{\prime}} \exp \left\{-i k\left[x_{0} R_{51}\left(s, s^{\prime}\right)+\theta_{0} R_{52}\left(s, s^{\prime}\right)\right]\right\} \\
=n_{b} \rho_{G}\left(p_{0}\right)\left[-\left.i k R_{51}\left(s, s^{\prime}\right) \frac{\partial x_{0}}{\partial p}\right|_{s^{\prime}}-\left.i k R_{52}\left(s, s^{\prime}\right) \frac{\partial \theta_{0}}{\partial p}\right|_{s^{\prime}}+\left.\frac{p_{0}}{\sigma_{p}^{2}}\left(1+u \frac{\partial z_{0}}{\partial p}\right)\right|_{s^{\prime}}\right] e^{-\left(k^{2} \epsilon_{0} / 2 \beta_{0}\right)\left[\beta_{0}^{2} R_{51}^{2}\left(s, s^{\prime}\right)+R_{52}^{2}\left(s, s^{\prime}\right)\right]} .
\end{gathered}
$$

This simplifies the kernel Eq. (31):

$$
\begin{aligned}
K\left(s, s^{\prime}\right)= & \frac{i k r_{e} n_{b}}{\gamma} C\left(s^{\prime}\right) Z\left(k C\left(s^{\prime}\right), s^{\prime}\right) \int d p_{0} \rho_{G}\left(p_{0}\right)\left[\left.R_{51}\left(s, s^{\prime}\right) \frac{\partial x_{0}}{\partial p}\right|_{s^{\prime}}+\left.R_{52}\left(s, s^{\prime}\right) \frac{\partial \theta_{0}}{\partial p}\right|_{s^{\prime}}+\left.\frac{i p_{0}}{k \sigma_{p}^{2}}\left(1+u \frac{\partial z_{0}}{\partial p}\right)\right|_{s^{\prime}}\right] \\
& \times e^{-\left(k^{2} \epsilon_{0} / 2 \beta_{0}\right)\left[\beta_{0}^{2} R_{51}^{2}\left(s, s^{\prime}\right)+R_{52}^{2}\left(s, s^{\prime}\right)\right]} .
\end{aligned}
$$


Integration over $d p_{0}$ gives finally:

$$
\begin{aligned}
K\left(s, s^{\prime}\right)= & \frac{i k r_{e} n_{b}}{\gamma} C\left(s^{\prime}\right) Z\left(k C\left(s^{\prime}\right), s^{\prime}\right)\left[\left.R_{51}\left(s, s^{\prime}\right) \frac{\partial x_{0}}{\partial p}\right|_{s^{\prime}}+\left.R_{52}\left(s, s^{\prime}\right) \frac{\partial \theta_{0}}{\partial p}\right|_{s^{\prime}}+\left.R_{56}\left(s, s^{\prime}\right)\left(1+u \frac{\partial z_{0}}{\partial p}\right)\right|_{s^{\prime}}\right] \\
& \times e^{-\left(k^{2} \epsilon_{0} / 2 \beta_{0}\right)\left[\beta_{0}^{2} R_{51}^{2}\left(s, s^{\prime}\right)+R_{52}^{2}\left(s, s^{\prime}\right)\right]-\left(k^{2} \sigma_{p}^{2} / 2\right) R_{56}^{2}\left(s, s^{\prime}\right)} .
\end{aligned}
$$

It is shown in Appendix A that the factor

$$
\left.R_{51}\left(s, s^{\prime}\right) \frac{\partial x_{0}}{\partial p}\right|_{s^{\prime}}+\left.R_{52}\left(s, s^{\prime}\right) \frac{\partial \theta_{0}}{\partial p}\right|_{s^{\prime}}+\left.R_{56}\left(s, s^{\prime}\right)\left(1+u \frac{\partial z_{0}}{\partial p}\right)\right|_{s^{\prime}}
$$

can be written as $C(s) R_{56}\left(s^{\prime} \rightarrow s\right)$, where

$$
R_{56}\left(s^{\prime} \rightarrow s\right)=-\int_{s^{\prime}}^{s} \frac{d s_{1}}{R\left(s_{1}\right)} \int_{s^{\prime}}^{s_{1}} \frac{d s_{2}}{R\left(s_{2}\right)} \sqrt{\beta\left(s_{1}\right) \beta\left(s_{2}\right)} \sin \left[\psi\left(s_{1}\right)-\psi\left(s_{2}\right)\right]=-\int_{s^{\prime}}^{s} d s_{1} \frac{D\left(s^{\prime}, s_{1}\right)}{R\left(s_{1}\right)},
$$

which depends only on the phase advance between radiation and observation points. The dispersion $D\left(s^{\prime}, s\right)$ in Eq. (38) satisfies the second equation of Eqs. (3) with the initial conditions $D\left(s^{\prime}, s^{\prime}\right)=0$ and $d D\left(s^{\prime}, s\right) /\left.d s\right|_{s=s^{\prime}}=0$. Using this representation, the kernel can be rewritten in the following form:

$$
K\left(s, s^{\prime}\right)=\frac{i k r_{e} n_{b}}{\gamma} C\left(s^{\prime}\right) C(s) Z\left(k C\left(s^{\prime}\right), s^{\prime}\right) R_{56}\left(s^{\prime} \rightarrow s\right) e^{-\left(k^{2} \epsilon_{0} / 2 \beta_{0}\right)\left[\beta_{0}^{2} R_{51}^{2}\left(s, s^{\prime}\right)+R_{52}^{2}\left(s, s^{\prime}\right)\right]-\left(k^{2} \sigma_{p}^{2} / 2\right) R_{56}^{2}\left(s, s^{\prime}\right)} .
$$

For the initial distribution function of the perturbation we choose $f_{k}\left(x_{0}, \theta_{0}, p_{0}, 0\right)=\left(n_{1, k}^{(0)} / n_{b}\right) f_{0}\left(x_{0}, \theta_{0}, p_{0}\right)$, where $n_{1, k}^{(0)}$ is the amplitude of the initial density perturbation with the wave number $k$. Then

$$
g_{k}^{(0)}(s)=n_{1, k}^{(0)} e^{-\left[C(s)^{2} k^{2} \epsilon_{0} / 2 \beta_{0}\right]\left[\beta_{0}^{2} R_{51}^{2}(s)+R_{52}^{2}(s)\right]-C(s)^{2} k^{2} \sigma_{p}^{2} R_{56}^{2}(s) / 2} .
$$

Equations (30), (35), and (40) describe beam stability for an arbitrary optics and initial longitudinal and transverse emittances, and form the basis for the numerical calculations described in the next section.

\section{NUMERICAL SOLUTION OF THE INTEGRAL EQUATION}

We applied the theory developed above to the analysis of stability in the latest design of the second LCLS compressor [14]. The bunch compressor consists of four dipole magnets of length $0.4 \mathrm{~m}$ with a bending radius of $12.2 \mathrm{~m}$. It is located at the point in the linac where the beam energy is equal to $4.54 \mathrm{GeV}$, and compresses the rms bunch length $\sigma_{l}$ from $195 \mu \mathrm{m}$ down to $23 \mu \mathrm{m}$. Other relevant parameters of the bunch compressor are uncorrelated rms relative energy spread at the entrance to the

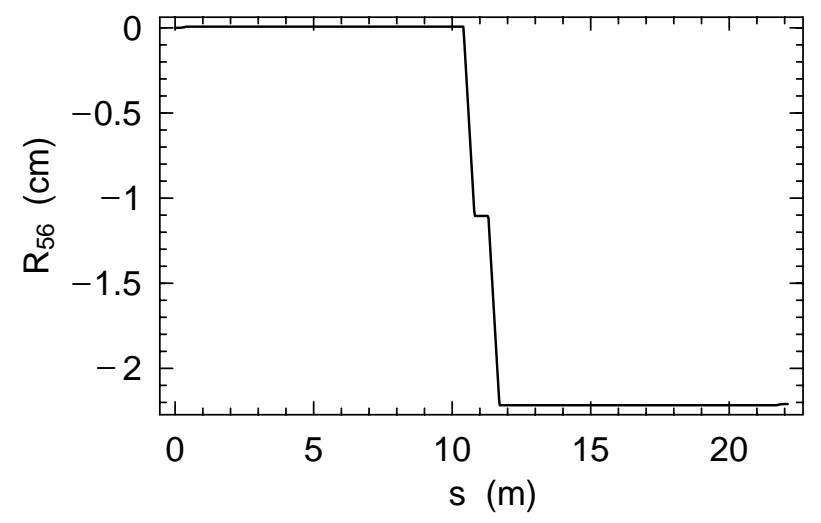

FIG. 1. Plot of $R_{56}$ for the LCLS bunch compressor. compressor $\sigma_{p}=3 \times 10^{-5}$, number of particles in the bunch $N=6.5 \times 10^{9}$, the normalized beam emittance $\epsilon=1 \mu \mathrm{m}$, and the energy chirp parameter $u$ in Eq. (2) is $-39.83 \mathrm{~m}^{-1}$. The calculated $R_{56}$ as a function of $s$ is shown in Fig. 1, and the plot of the dispersion function $D(s)$ is shown in Fig. 2.

We calculated the microbunching effect in the bunch compressor by numerically solving Eq. (30) with the kernel given by Eq. (39). The numerical method used discretization of the Volterra equation on a mesh, typically with 400-600 points, and approximation of an integral by a sum using a trapezoidal quadrature rule [15].

We assumed that the initial distribution function is given by Eq. (9) and the initial perturbation is defined by Eq. (40). The linear bunch density $n_{b}$ was calculated

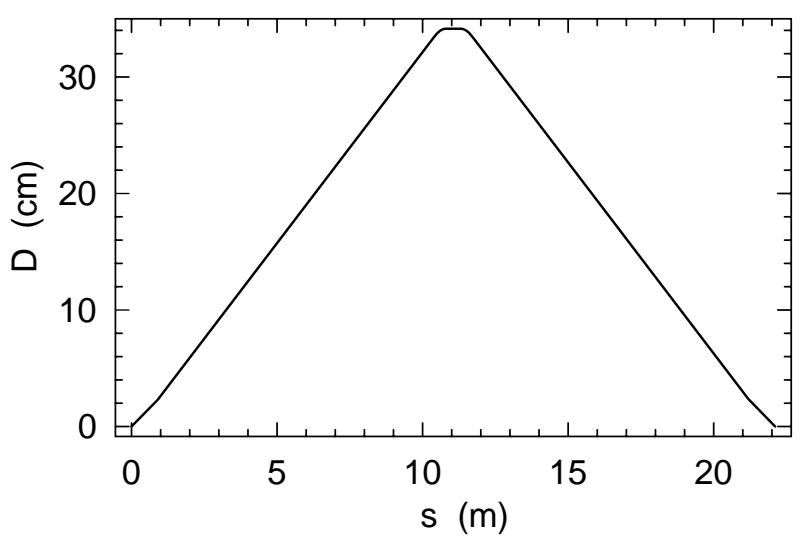

FIG. 2. Plot of the dispersion function for the LCLS bunch compressor. 


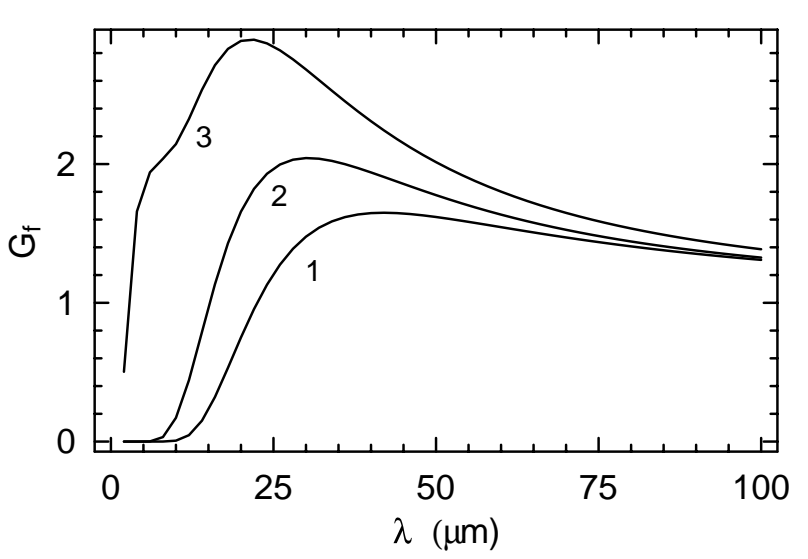

FIG. 3. Amplification factor $G_{f}$ as a function of wavelength $\lambda$ of the perturbation at the compressor entrance for various beam emittance and energy spread: (1) $\sigma_{p}=$ $3.0 \times 10^{-5}, \epsilon=1 \mu \mathrm{m} ;$ (2) $\sigma_{p}=3.0 \times 10^{-5}, \epsilon=0$; and (3) $\sigma_{p}=3.0 \times 10^{-6}, \epsilon=1 \mu \mathrm{m}$.

based on the beam current of $I=4 \mathrm{kA}$ after compression. The current $I$ is related to $n_{b}$ at the entrance to the chicane by the following formula:

$$
n_{b}=\frac{1}{C r_{e}} \frac{I}{I_{A}}
$$

where $C$ is the compression factor for the chicane and $I_{A}$ is the Alfvén current, $I_{A}=m c^{3} / e=17 \mathrm{kA}$. For $C=8.33$, we find $n_{b}=10^{11} \mathrm{~cm}^{-1}$.

At the entrance to the compressor, an initial density perturbation $n_{1, k}^{(0)}$ with the wavelength $\lambda=2 \pi / k$ has been specified and the ratio $\left|n_{1, k}(z, s)\right| / n(s)$ has been calculated throughout the compressor, where $n_{1, k}$ is given by Eq. (29) (note that the absolute value $\left|n_{1, k}(s, z)\right|$ is a function of $s$ only). The amplification factor $G(s)$ for the density perturbations is defined as

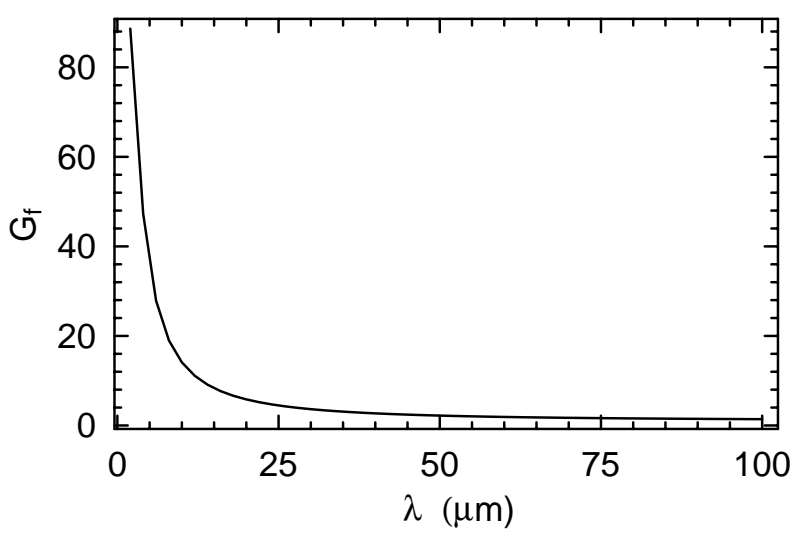

FIG. 4. Amplification factor $G_{f}$ as a function of wavelength for the zero beam emittance and $\sigma_{p}=3.0 \times 10^{-6}$.

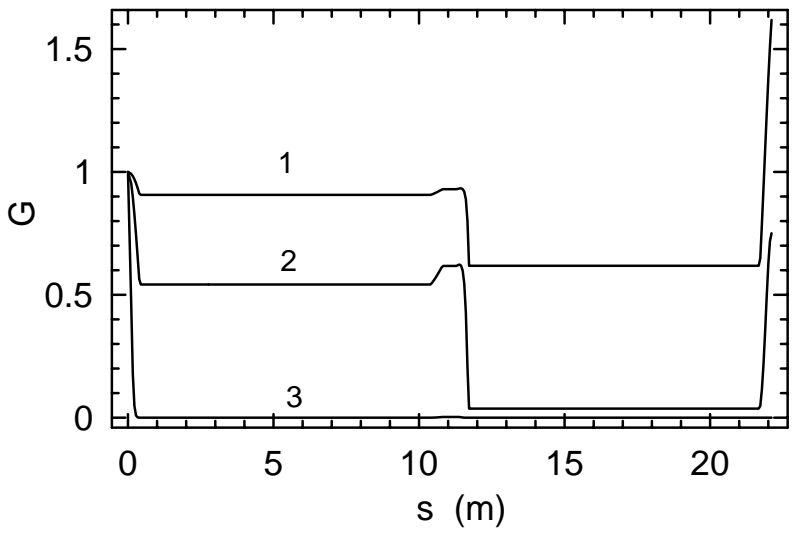

FIG. 5. Amplification factor $G(s)$ for $\epsilon=1 \mu \mathrm{m}, \sigma_{p}=3.0 \times$ $10^{-5}$ for three values of $\lambda$ : (1) $\lambda=50 \mu \mathrm{m}$, (2) $\lambda=20 \mu \mathrm{m}$, and (3) $\lambda=5 \mu \mathrm{m}$.

$$
G(s)=\frac{\left|n_{1, k}(s, z)\right|}{C(s) n_{1, k}^{(0)}}
$$

which characterizes the growth of the relative density perturbation of the beam [the linear beam density $n(s)$ increases by a factor of 8.3 at the end of the compressor].

To illustrate the dependence of the amplification factor on the energy spread and the beam emittance, in addition to the nominal LCLS beam parameters listed above, we performed calculations for 10 times smaller energy spread $\sigma_{p}=3.0 \times 10^{-6}$ and zero beam emittance. The amplification factor at the end of the chicane $G_{f}$ as a function of the initial wavelength of the modulation is shown in Figs. 3 and 4 for four cases of various beam emittance and energy spread. We see that both a larger energy spread and the finite beam emittance in Fig. 3 result in the strong suppression of the growth relative to the case $\sigma_{p}=3.0 \times 10^{-6}$, $\epsilon=0$ shown in Fig. 4 .

The profile of the amplification factor $G(s)$ for several different wavelengths inside the bunch compressor is shown in Figs. 5-7.

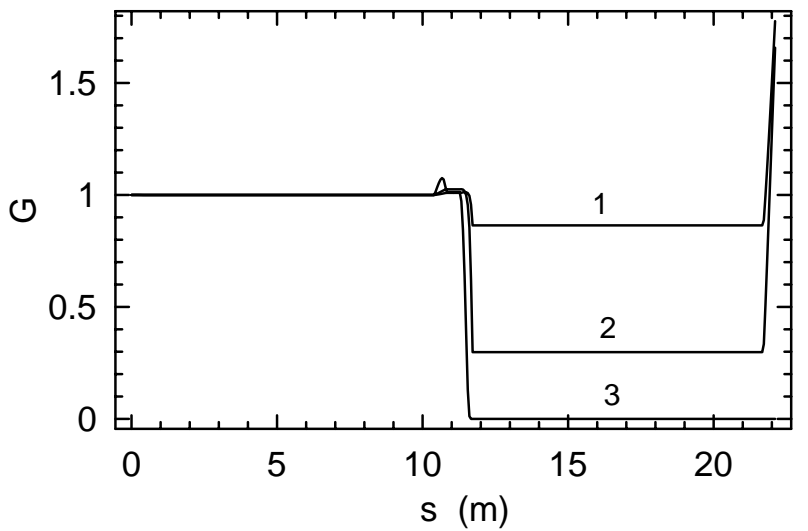

FIG. 6. Amplification factor $G(s)$ for $\epsilon=0, \sigma_{p}=3.0 \times$ $10^{-5}$ for three values of $\lambda$ : (1) $\lambda=50 \mu \mathrm{m}$, (2) $\lambda=20 \mu \mathrm{m}$, and (3) $\lambda=5 \mu \mathrm{m}$. 


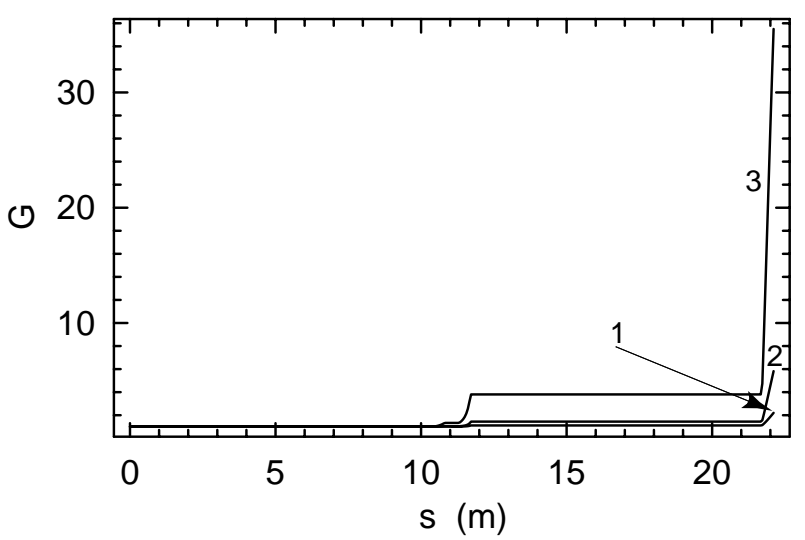

FIG. 7. Amplification factor $G(s)$ for $\epsilon=0, \sigma_{p}=3.0 \times$ $10^{-6}$ for three values of $\lambda$ : (1) $\lambda=50 \mu \mathrm{m}$, (2) $\lambda=20 \mu \mathrm{m}$, and (3) $\lambda=5 \mu \mathrm{m}$.

\section{CONCLUSION}

In this paper we developed a linear theory describing self-induced microbunching of a beam in a magnetic bunch compressor. The microbunching results from the microwave instability driven in a self-consistent way by the coherent synchrotron radiation of the short-wavelength modulation. Solving the linearized Vlasov equation with longitudinal and radial degrees of freedom has provided a new description of the effect of CSR in bunch compressors. Including the transverse motion has facilitated a proper description of the bunching resulting from the CSR produced energy deviations. Also, the Landau damping due to the transverse emittance has proved to be an important stabilizing influence. The approach we presented here is complementary to numerical simulation of the problem since the approximations made are different, as are the sources of numerical error.

The techniques developed in this paper are also applicable to investigation of CSR induced instabilities in storage rings. A dispersion relation describing the growth rate of the microwave instability in a storage ring is derived in Appendix B using the smooth approximation for the transverse optics. A detailed analysis of the microwave instability driven by CSR in storage rings will be given in a separate paper.

Numerical calculation for the latest design of the LCLS bunch compressor shows that an initial density perturbation with a wavelength in the range of $30-100 \mu \mathrm{m}$ is slightly amplified by a factor less than 2 .

We emphasize here that the wake Eq. (11) used in this paper may not be applicable for very short wavelength. Indeed, this wake was derived for a bunch that is infinitely thin in the transverse direction and assumes that all particles in the cross section of the bunch radiate coherently. However, the transverse coherence length $l_{\perp} \sim \lambda^{2 / 3} R^{1 / 3}$ decreases with the wavelength and at some point becomes smaller than the transverse dimension of the beam. For such wavelength, one has to use a wake that takes into account the transverse dimension of the beam.

\section{ACKNOWLEDGMENTS}

We thank P. Emma who calculated the LCLS bunch compressor optical functions. This work was supported by the U.S. Department of Energy Contracts No. DE-AC0376SF00515 and No. DE-AC02-98CH10886.

\section{APPENDIX A: DISCUSSION OF EQ. (37)}

Let us consider the factor

$$
\begin{aligned}
F \equiv & \left.R_{51}\left(s, s^{\prime}\right) \frac{\partial x_{0}}{\partial p}\right|_{s^{\prime}}+\left.R_{52}\left(s, s^{\prime}\right) \frac{\partial \theta_{0}}{\partial p}\right|_{s^{\prime}} \\
& +\left.R_{56}\left(s, s^{\prime}\right)\left(1+u \frac{\partial z_{0}}{\partial p}\right)\right|_{s^{\prime}}
\end{aligned}
$$

appearing in Eq. (36) and rewrite it in a form that clarifies its physical meaning. The dispersion specified in Eq. (5) can be written as

$$
\begin{aligned}
D(s)=\sqrt{\beta(s)}[ & -\sqrt{\beta_{0}} R_{51}(s) \sin \psi(s) \\
& \left.+\frac{1}{\sqrt{\beta_{0}}} R_{52}(s) \cos \psi(s)\right] .
\end{aligned}
$$

Substituting Eq. (A2) into Eq. (20), we find

$$
\begin{gathered}
\left.\frac{\partial x_{0}}{\partial p}\right|_{s^{\prime}}=-R_{52}\left(s^{\prime}\right),\left.\quad \frac{\partial \theta_{0}}{\partial p}\right|_{s^{\prime}}=-R_{51}\left(s^{\prime}\right), \\
\left.\frac{\partial z_{0}}{\partial p}\right|_{s^{\prime}}=-R_{56}\left(s^{\prime}\right) .
\end{gathered}
$$

Now, using Eqs. (A3) in Eq. (A1), the factor $F$ can be rewritten in the form

$$
F=C(s) R_{56}\left(s^{\prime} \rightarrow s\right),
$$

where the compression factor $C(s)$ was defined earlier in Eq. (26), and

$$
\begin{aligned}
R_{56}\left(s^{\prime} \rightarrow s\right)= & -\int_{s^{\prime}}^{s} \frac{d s_{1}}{R\left(s_{1}\right)} \sqrt{\beta\left(s_{1}\right)} \int_{s^{\prime}}^{s_{1}} \frac{d s_{2}}{R\left(s_{2}\right)} \\
& \times \sqrt{\beta\left(s_{2}\right)} \sin \left[\psi\left(s_{1}\right)-\psi\left(s_{2}\right)\right] .
\end{aligned}
$$

The physical meaning of the quantity defined in Eq. (A5) is clear. If at position $s^{\prime}$ the energy is changed by $\Delta p$, the increment in path length from $s^{\prime}$ to $s$ is $R_{56}\left(s^{\prime} \rightarrow s\right) \Delta p$.

\section{APPENDIX B: DISPERSION RELATION FOR STORAGE RING}

For a storage ring, the chirp $u$ vanishes and the compression factor is equal to unity, $C(s) \equiv 1$. The dispersion 
function $D(s)$ is defined as a periodic function of $s$ and is given by the following equation:

$$
\begin{aligned}
D(s)= & \frac{\sqrt{\beta(s)}}{2 \sin (\pi \nu)} \int_{s}^{s+L} \frac{d s^{\prime}}{R\left(s^{\prime}\right)} \\
& \times \sqrt{\beta\left(s^{\prime}\right)} \cos \left[\psi(s)-\psi\left(s^{\prime}\right)+\pi \nu\right],
\end{aligned}
$$

where $L$ is the ring circumference and $\nu$ is the horizontal tune.

Our derivation of the integral equation (30) remains valid for the storage ring, with the kernel given by either Eq. (35) or (36), if the matrix element $R_{56}$ in Eq. (4) is calculated using expression (B1). Notice that since $u=0$, Eqs. (32) take the form

$$
\begin{aligned}
& R_{51}\left(s, s^{\prime}\right)=R_{51}(s)-R_{51}\left(s^{\prime}\right), \\
& R_{52}\left(s, s^{\prime}\right)=R_{52}(s)-R_{52}\left(s^{\prime}\right), \\
& R_{56}\left(s, s^{\prime}\right)=R_{56}(s)-R_{56}\left(s^{\prime}\right) .
\end{aligned}
$$

Using Eqs. (4) it easy to show that $R_{51}\left(s, s^{\prime}\right), R_{52}\left(s, s^{\prime}\right)$, $R_{56}\left(s, s^{\prime}\right)$, and hence the kernel $K\left(s, s^{\prime}\right)$ do not depend on the choice of the initial position $s=0$ in the ring.

Specifically, for the ring, the kernel Eq. (36) takes the form

$$
K\left(s, s^{\prime}\right)=\frac{i k r_{e} n_{b}}{\gamma} Z\left(k, s^{\prime}\right) R_{56}\left(s^{\prime} \rightarrow s\right) e^{-\left(k^{2} \epsilon_{0} / 2 \beta_{0}\right)\left[\beta_{0}^{2} R_{51}^{2}\left(s, s^{\prime}\right)+R_{52}^{2}\left(s, s^{\prime}\right)\right]-\left(k^{2} \sigma_{p}^{2} / 2\right) R_{56}^{2}\left(s, s^{\prime}\right)} .
$$

The integral equation for the ring can be simplified if one uses a smooth approximation for the ring lattice with a tune $\nu$. In this approximation, the bending radius $R$ is constant, $\beta=R / \nu, \psi=\nu s / R, D=R / \nu^{2}, \alpha=0, D^{\prime}=0$, and $\epsilon_{0}=\sigma_{x}^{2} \nu / R$, where $\sigma_{x}$ is the horizontal rms size of the beam. Note also that in this approximation the slip factor for the lattice $\eta$ is equal to $1 / \nu^{2}$. One can show that

$$
\begin{aligned}
\beta R_{51}^{2}\left(s, s^{\prime}\right)+\frac{1}{\beta} R_{52}^{2}\left(s, s^{\prime}\right) & =\frac{2 R}{\nu^{3}}\left[1-\cos \left(\frac{\nu\left(s-s^{\prime}\right)}{R}\right)\right], \\
R_{56}\left(s, s^{\prime}\right) & =-\frac{1}{\nu^{2}}\left(s-s^{\prime}\right), \\
R_{56}\left(s^{\prime} \rightarrow s\right) & =-\frac{1}{\nu^{2}}\left[\left(s-s^{\prime}\right)-\frac{R}{\nu} \sin \left(\frac{\nu\left(s-s^{\prime}\right)}{R}\right)\right] .
\end{aligned}
$$

The kernel Eq. (B3) takes the form:

$$
K\left(s, s^{\prime}\right)=K_{1}\left(s-s^{\prime}\right) \equiv-i \frac{r_{e} n_{b}}{\nu^{2} \gamma} k Z(k)\left[-\frac{R}{\nu} \sin \left(\frac{\nu\left(s-s^{\prime}\right)}{R}\right)+s-s^{\prime}\right] e^{\left.-\left(k \sigma_{x} / \nu\right)^{2}\left\{1-\cos \left[\nu\left(s-s^{\prime}\right) / R\right)\right]\right\}-1 / 2\left(k \sigma_{p} / \nu^{2}\right)^{2}\left(s-s^{\prime}\right)^{2}} \text {. }
$$

This kernel depends only on the difference $s-s^{\prime}$, and one can use the Laplace transform to cast it into an algebraic for the Laplace image $\hat{g}_{k}(\mu)$,

$$
\hat{g}_{k}(\mu)=\int_{0}^{\infty} d s g_{k}(s) e^{-\mu s}
$$

of the function $g_{k}(s)$. Applying the Laplace transform to Eq. (30) yields

$$
\hat{g}_{k}(\mu)=\frac{\hat{g}_{k}^{(0)}(\mu)}{1-\hat{K}(\mu)},
$$

where $\hat{K}(\mu)$ is the Laplace transform of the kernel $K_{1}(s)$,

$$
\hat{K}(\mu)=\int_{0}^{\infty} d s e^{-\mu s} K_{1}(s)
$$

and

$$
\hat{g}_{k}^{(0)}(\mu)=\int_{0}^{\infty} d s g_{k}^{(0)}(s) e^{-\mu s}
$$

The dispersion relation is defined by the zeros of the denominator, $\hat{K}(\mu)=1$. For the kernel given by Eq. (B5), this equation gives

$$
\begin{aligned}
1= & -\frac{i r_{e} n_{b}}{\nu^{2} \gamma} k Z(k) \int_{0}^{\infty} d s e^{-\mu s}\left[s-\frac{R}{\nu} \sin \frac{\nu s}{R}\right] \\
& \times e^{-\left(k \sigma_{x} / \nu\right)^{2}[1-\cos (\nu s / R)]-\left(k \sigma_{p} / \nu^{2}\right)^{2} s^{2} / 2} .
\end{aligned}
$$

[1] H. H. Braun, R. Corsini, L. Groening, F. Zhou, A. Kabel, T. Raubenheimer, R. Li, and T. Limberg, Phys. Rev. ST Accel. Beams 3, 124402 (2000).

[2] P. Emma and R. Brinkmann, in Proceedings of the 1997 Particle Accelerator Conference, Vancouver, Canada (IEEE, Piscataway, NJ, 1997).

[3] S. Heifets and G. Stupakov, SLAC Technical Report No. SLAC-PUB-8761, 2001.

[4] M. Borland and P. Emma (private communication).

[5] The LCSL Design Study Group, SLAC Report No. SLAC-R-521, 1998.

[6] T. Limberg, P. Piot, and E. A. Schneidmiller, Nucl. Instrum. Methods Phys. Res., Sect. A 475, 353 (2001).

[7] E. L. Saldin, E. A. Schneidmiller, and M. V. Yurkov, Nucl. Instrum. Methods Phys. Res., Sect. A 483, 516 (2002).

[8] S. Heifets and G. Stupakov, SLAC Technical Report No. SLAC-PUB-8988, 2001. 
[9] E. L. Saldin, E. A. Schneidmiller, and M. V. Yurkov DESY Technical Report No. DESY-TESLA-FEL-2002-02, 2002.

[10] E. L. Saldin, E. A. Schneidmiller, and M. V. Yurkov, Nucl. Instrum. Methods Phys. Res., Sect. A 398, 373 (1997).

[11] Note that in the standard notations the transport elements $r_{51}, r_{52}$, and $r_{56}$ are defined so that $z(s)=z_{0}+r_{56}(s) p+$ $r_{51}(s) x(0)+r_{52}(s) \theta(0)$. Because $x_{0}=x(0)$ and $\theta_{0}=$ $\theta(0)+x(0) \alpha(0) / \beta(0)$, our functions $R_{51}, R_{52}$, and $R_{56}$ are related to these standard elements of the transport matrix by the equations $R_{56}=r_{56}, R_{52}=r_{52}$, and $R_{51}=$ $r_{51}-r_{52} \alpha(0) / \beta(0)$.

[12] J. B. Murphy, S. Krinsky, and R. L. Gluckstern, in Proceedings of the IEEE Particle Accelerator Conference, Dallas, 1995 (IEEE, Piscataway, NJ, 1996).

[13] Y.S. Derbenev, J. Rossbach, E. L. Saldin, and V. D. Shiltsev, DESY FEL Report No. TESLA-FEL 95-05, 1995.

[14] P. Emma (private communication).

[15] W. H. Press, S. A. Teukolsky, W. T. Vetterling, and B.P. Flannery, Numerical Recipes in $C$ (Cambridge University Press, New York, 1995), 2nd ed. 